01,07

\title{
Температурная зависимость автоволновых характеристик локализованной пластичности
}

\author{
(С) Л.Б. Зуев, С.А. Баранникова, С.В. Колосов, А.М. Никонова \\ Институт фризики прочности и материаловедения СО РАН, \\ Томск, Россия \\ E-mail: lbz@ispms.tsc.ru
}

Поступила в Редакцию 12 августа 2020 г.

В окончательной редакции 12 августа 2020 г.

Принята к публикации 3 сентября 2020 г.

\begin{abstract}
Рассмотрено поведение автоволн локализованного пластического течения в сплаве $\mathrm{Fe}-\mathrm{Ni}-\mathrm{Cr}$ при температурах $143 \leq T \leq 420 \mathrm{~K}$. Изучен температурный ход скорости распространения автоволны. Установлено, что для области низких температур выполняются обратная пропорциональность скорости автоволны коэффициенту деформационного упрочнения и квадратичный закон дисперсии. Установлена температурная независимость упругопластического инварианта деформации.
\end{abstract}

Ключевые слова: деформация, пластичность, автоволны, длина автоволны, скорость автоволны, локализация, низкие температуры, дислокации.

DOI: 10.21883/FTT.2021.01.50397.169

\section{1. Введение}

Экспериментальные исследования кинетики развития пластической деформации твердых тел [1-3] показали, что в ходе деформационного процесса формируются различные моды автоволн локализованного пластического течения, закономерно меняющиеся при изменении закона деформационного упрочнения. Автоволны характеризуются длиной $\lambda$, периодом $T$ и скоростью распространения $V_{a w}=\lambda / T$. Их скорость обратно пропорциональна коэффициенту деформационного упрочнения $V_{a w} \sim \theta^{-1}$, а закон дисперсии имеет квадратичный характер $\omega \sim 1+k^{2}$, где $\omega=2 \pi / T-$ частота, а $k=2 \pi / \lambda-$ волновое число.

Характеристики автоволны $\lambda$ и $V_{a w}$ связаны с межплоскостным расстоянием $\chi$ и скоростью поперечных упругих волн $V_{t}$ безразмерным отношением

$$
\frac{\lambda V_{a w}}{\chi V_{t}}=\hat{Z} \approx \frac{1}{2},
$$

справедливым для стадий линейного деформационного упрочнения и названным упругопластическим инвариантом деформации. Природа этого соотношения определяется взаимной обусловленностью процессов, одновременно протекающих в фононной и деформационной подсистемах пластически деформируемой среды $[1,2]$. Выполнимость инварианта (1) была установлена на основании опытов по деформации металлов, щелочно-галоидных кристаллов и горных пород, a также в экспериментах по ползучести и по измерениям пробегов индивидуальных дислокаций в монокристаллах [1]. Инвариант указывает на связь двух разномасштабных пространственно-временны́х процессов, реализующихся одновременно в деформируемой среде: перераспределения упругих деформаций (масштаб $\chi$, скорость $V_{t}$ ) и перераспределения локализованной пластической деформации (масштаб $\lambda$, скорость $\left.V_{a w}\right)$. Он играет важную роль в механике пластической деформации, а следствия из него [1,2] описывают наиболее важные закономерности пластической деформации.

В исследованиях [1-3] выполнение зависимостей $V_{a w} \sim \theta^{-1}$ для скорости и $\omega \sim 1+k^{2}$ для дисперсии, а также инварианта (1) было проверено для деформации при $300 \mathrm{~K}$. Задачей настоящей работы был анализ температурных зависимостей характеристик автоволновых деформационных процессов.

\section{2. Исследованный материал и экспериментальная методика}

Эксперименты были выполнены на поликристаллическом ГЦК-сплаве $\mathrm{Fe}-18$ wt.\% $\mathrm{Cr}-10$ wt.\% Ni с размером зерна $\sim 12.5 \mu \mathrm{m}$. Образцы с размерами рабочей части $40 \times 5 \times 2 \mathrm{~mm}$ растягивались на испытательной машине „Instron-1185“ со скоростью $3.3 \cdot 10^{-4} \mathrm{~s}^{-1}$ при температурах $420,296,266,243,213,143 \mathrm{~K}$, то есть, при $T \leq \theta_{D}$, где $\theta_{D} \approx 420 \mathrm{~K}$ - температура Дебая для железа [4]. Температура испытания задавалась скоростью продувки рабочей камеры, где находился образец при испытании, парами азота из сосуда Дьюара и контролировалась хромель-алюмелевой термопарой, спай которой контактировал с образцом. Скорость продувки парами азота регулировалась нагревательным элементом, смонтированным внутри сосуда Дьюара.

Автоволновая мода, возникающая на стадии линейного деформационного упрочнения (фазовая автоволна) 

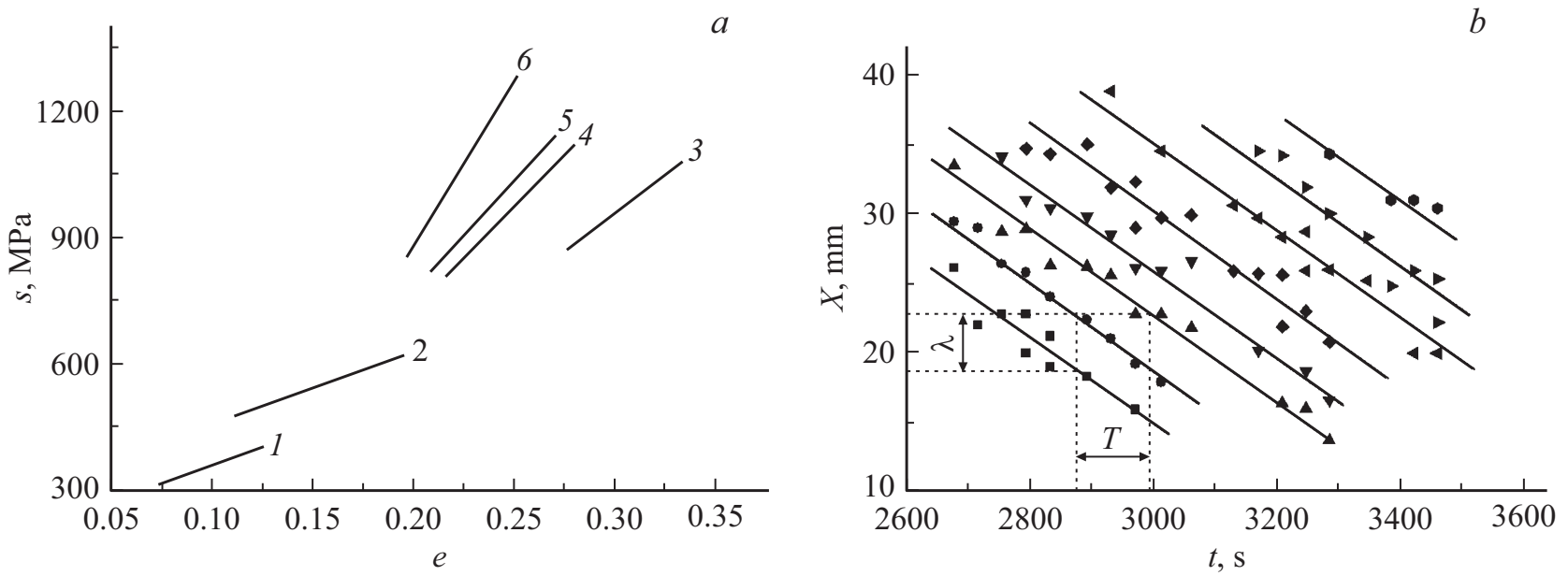

Рис. 1. Участки линейного деформационного упрочнения в координатах $e-s: 1-420,2-296,3-266,4-243,5-$ $213,6-143 \mathrm{~K}(a)$. Пример $X-t$ кинетической диаграммы деформации: $T=266 \mathrm{~L}: X-$ координата деформационной полосы, $t$ - время $(b)$.

наиболее устойчива [1], и при анализе результатов внимание было обращено на автоволновые характеристики деформационного процесса на этой стадии. Для выделения линейных стадий записанные первоначально в координатах „напряжение $\sigma$-деформация $\varepsilon^{\text {“ }}$ индикаторные кривые преобразовывались в зависимости истинных напряжений $\mathrm{s}$ от истинных деформаций $e$ [5]. Далее на кривых $s(e)$ выделялись участки, для которых $s \sim e$, а коэффициент деформационного упрочнения, нормированный на упругий модуль $E, \theta=E^{-1} d s / d e=$ const, как показано на рис. $1, a$.

Для визуализации автоволновых картин непосредственно в ходе механических испытаний при пониженных температурах методом двухэкспозиционной спеклфотографии [6] восстанавливались поля векторов смещений на поверхности образцов $\mathbf{r}(x, y)$. Дифференцированием этих полей по координатам $x$ и $y$ вычислялись компоненты тензора пластической дисторсии для всех точек наблюдаемой плоской поверхности и строились их распределения по образцу для разных моментов времени. Характеристики автоволн локализованной пластичности $\lambda$ и $V_{a w}$, постоянные при линейном деформационном упрочнении, оценивались по $X-t$ диаграммам, построенным на основе анализа распределений продольной компоненты тензора дисторсии $\varepsilon_{x x}$, как показано на рис. $1, b$.

\section{3. Результаты экспериментов и их интерпретация}

Как следует из табл. 1, механические свойства сплава (временное сопротивление $\sigma_{B}$, условный предел текучести $\sigma_{02}$, относительное удлинение до разрыва $\delta$ и коэффициент деформационного упрочнения $\theta$ ) существенно зависят от температуры. Важным аспектом настоящего исследования является анализ возможной
Таблица 1. Механические характеристики исследованного сплава

\begin{tabular}{c|c|c|c|c|c|c}
\hline$T, \mathrm{~K}$ & $\sigma_{B}, \mathrm{MPa}$ & $\sigma_{02}, \mathrm{MPa}$ & $\delta$ & $\theta \cdot 10^{3}$ & $\lambda \cdot 10^{3}, \mathrm{~m}$ & $V_{a w} \cdot 10^{5}, \mathrm{~m} / \mathrm{s}$ \\
\hline 420 & 454 & 194 & 0.4 & 8 & 4.5 & 7.6 \\
\hline 296 & 790 & 269 & 0.7 & 9 & 5.3 & 4.7 \\
\hline 266 & 860 & 292 & 0.55 & 10.9 & 4.9 & 4.1 \\
\hline 243 & 955 & 287 & 0.44 & 21.5 & 3.5 & 3.7 \\
\hline 213 & 988 & 293 & 0.43 & 26.2 & 4.2 & 3.1 \\
\hline 143 & 1129 & 291 & 0.37 & 38.8 & 4.6 & 2.5
\end{tabular}

температурной зависимости инварианта (1) и входящих в него величин. Так как величины $\chi$ и $V_{t}$ с температурой меняются слабо [7], а $\lambda$, как сказано выше, постоянна в исследованном температурном интервале, то специальный интерес представляет температурная зависимость скорости распространения автоволн локализованной пластичности $V_{a w}$, входящей в уравнение инварианта (1).

\section{1. Скорость распространения автоволн}

Экспериментально полученная температурная зависимость скорости распространения автоволн $V_{a w}(T)$ показана на рис. 2, $a, b$. Длина автоволны в этом интервале в пределах точности эксперимента не зависит от температуры и составляет $\lambda=4.5 \pm 0.25 \mathrm{~mm}$. Это позволяет считать зависимости $V_{a w}(T)$ и $\lambda V_{a w}(T)$ эквивалентными.

По данным о величинах скоростей фронтов локализованной пластичности на стадиях линейного деформационного упрочнения и соответствующих коэффициентов деформационного упрочнения (табл. 1) был установлен 

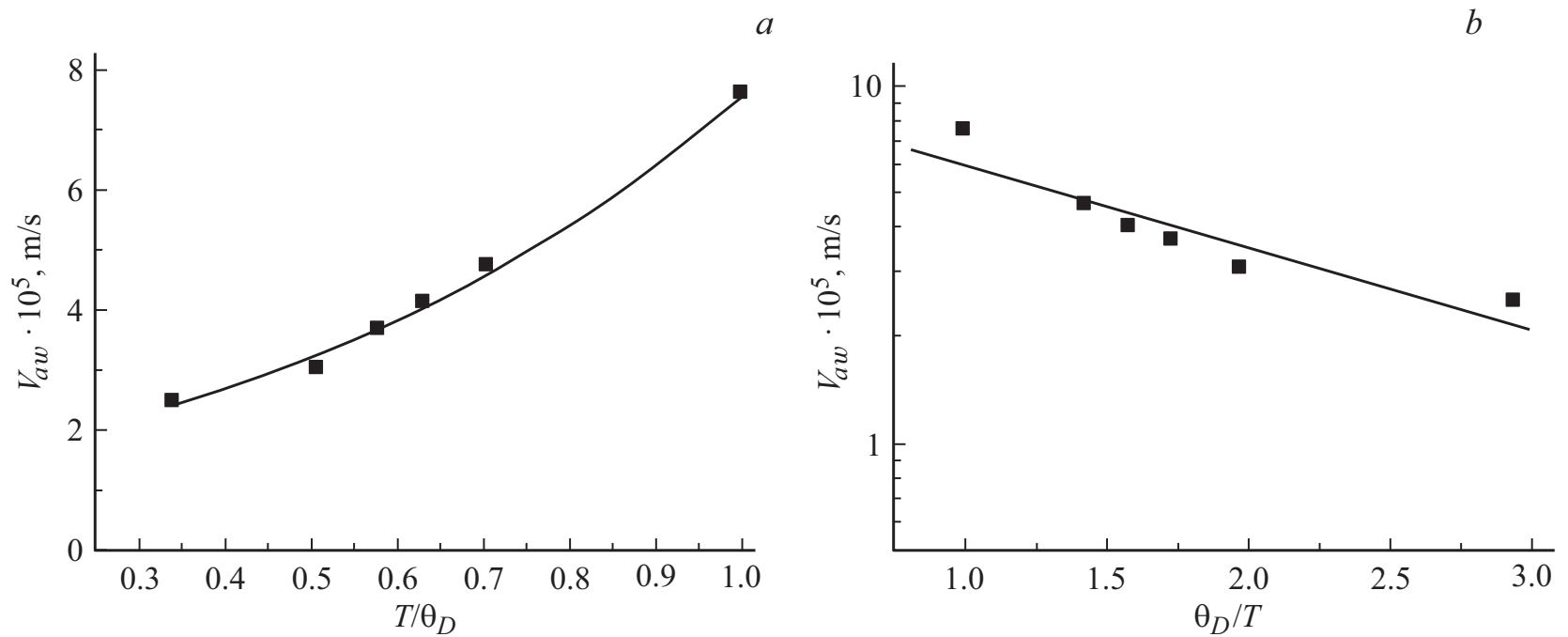

Рис. 2. Температурная зависимость скорости распространения автоволн локализованной пластичности в координатах $T-V_{a w}(a)$ и $T^{-1}-\ln V_{a w}(b)$.


Рис. 3. Скорость распространения автоволн локализованной пластичности в исследованном сплаве при разных температурах (a) и для всех исследованных металлов $(b)$ как функция коэффициента деформационного упрочнения.

вид функции $V_{a w}(\theta)$ при пониженных температурах. Рис. 3, $a$ показывает, что

$$
V_{a w}(\theta)=V_{0}+\frac{\Xi}{\theta} \sim \theta^{-1}
$$

Как сказано выше, аналогичное соотношение было установлено по результатам исследований автоволновой пластичности материалов при $300 \mathrm{~K}[1,2]$. Значения констант $V_{0}=1.8 \cdot 10^{-5} \mathrm{~m} / \mathrm{s}$ и $\Xi=9.8 \cdot 10^{-7} \mathrm{~m} / \mathrm{s}$ в уравнении (2) близки к найденным ранее общим для исследованных ранее материалов значениям, полученным при $T \approx 300 \mathrm{~K}$. Измеренные для низких температур скорости распространения автоволн локализованной пластичности хорошо укладываются на обобщающий график зависимости $V_{a w}(\theta)$, как это показано на рис. $3, b$.

\section{2. Дисперсионное соотношение для автоволн}

По полученным распределениям компонент тензора пластической дисторсии были определены частота $\omega$ и волновое число $k$ для автоволн локализованной пластичности при разных температурах. Как оказалось, связывающее их дисперсионное уравнение $\omega(k)$ имеет квадратичную форму

$$
\omega(k)=\omega_{0}+\alpha\left(k-k_{0}\right)^{2} \sim k^{2},
$$

найденную ранее [2] и характерную для ряда нелинейных волн [8].

В уравнении (3) $\omega_{0}, k_{0}$ и $\alpha$-эмпирические константы, значения которых даны в табл. 2. Их сравнение с ранее полученными данными, представленными на рис. 4, $a$ и в табл. 2, демонстрирует хорошее согласие с результа- 

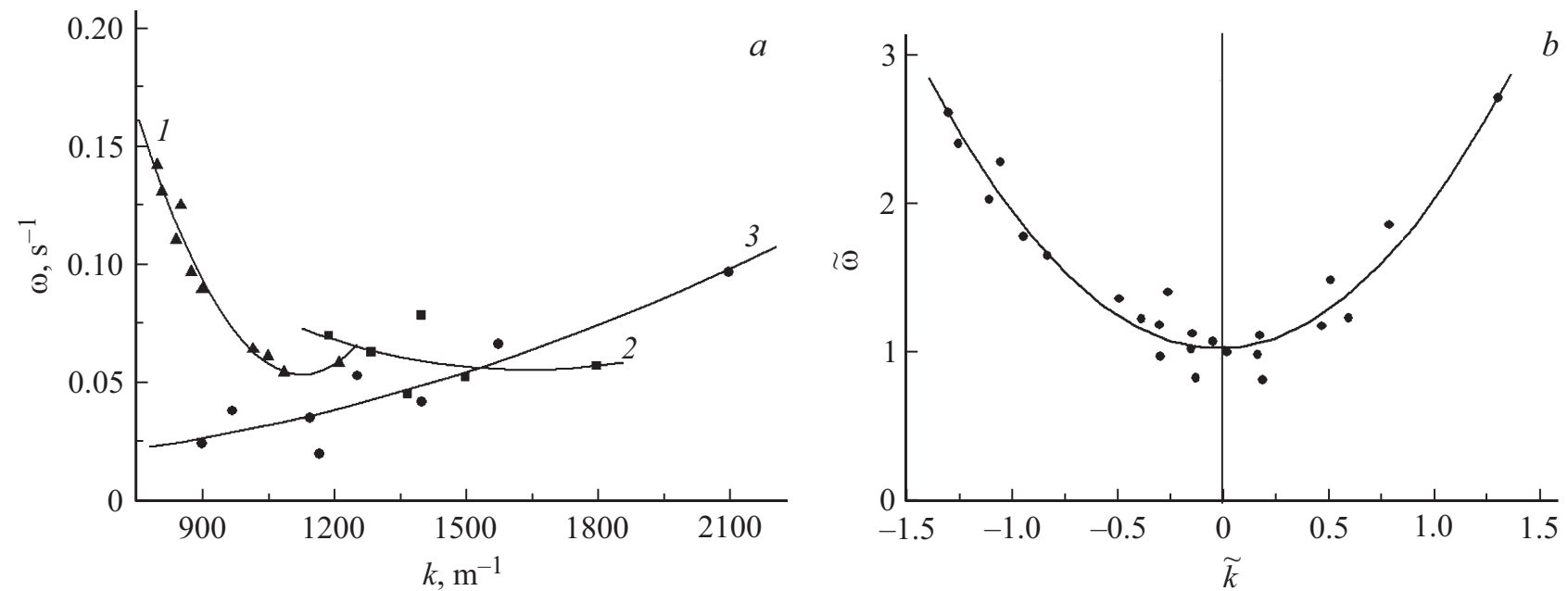

Рис. 4. Дисперсионное соотношение для скорости автоволн локализованной пластичности 1 - поликристаллы $\mathrm{Al}, 2$ - поликристаллы $\mathrm{Fe}$ (настоящее исследование), 3 - монокристаллы $\mathrm{Fe})(a)$; то же в обобщенном виде $(b)$.

тами для монокристаллов сплава $\mathrm{Fe}-\mathrm{Ni}-\mathrm{Cr}$ и поликристаллов Al. Из рис. 4, $b$ следует, что низкотемпературные значения частоты $\omega$ и волнового числа $k$ автоволн укладываются на описанную ранее в [2] обобщенную кривую $\tilde{\omega}(\tilde{k})$, где $\tilde{\omega}$ и $\tilde{k}-$ обезразмеренные частота и волновое число соответственно.

\section{3. Термоактивационный анализ автоволновых характеристик}

Из рис. $2, b$ следует, что зависимость $V_{a w}(T)$ для $1 \leq \theta_{D} / T \leq 3$ спрямляется в координатах $\ln V_{a w}-T^{-1}$, т. е., может быть аппроксимирована соотношением

$$
\left(\lambda V_{a w}\right) \sim V_{a w}=V^{*} \exp \left(-\frac{H}{k_{\mathrm{B}} T}\right),
$$

характерным для термически активированных процессов [9], где $H \approx U-\gamma \sigma-$ энтальпия активации, $k_{\mathrm{B}}-$ постоянная Больцмана, $U-$ потенциальный барьер, $\gamma$ - активационный объем, а $V^{*}$ - константа. По данным рис. 5, где выбранные для расчета напряжения отвечают условию $e=$ const, получаем $U \approx 0.06 \mathrm{eV}$, а $\gamma \approx 8.5 b^{3}$, где $b \approx 0.2 \mathrm{~nm}-$ вектор Бюргерса дислокаций в ГЦК-Fe.

Такие низкие значения активационных параметров означают, что автоволны локализованной пластичности

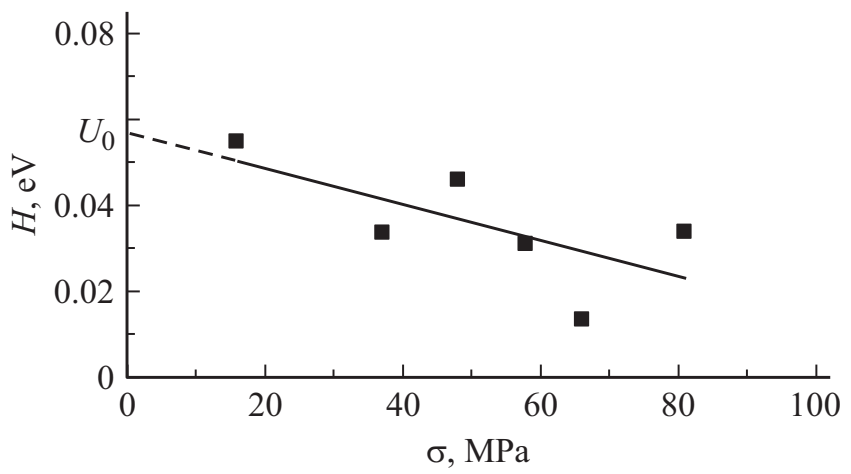

Рис. 5. К определению термоактивационных параметров развития автоволн локализованной пластичности по температурной зависимости скорости автоволн.

могут спонтанно порождаться в кристаллах при всех режимах деформирования, что и наблюдается в действительности [1]. На это же указывает и низкое значение константы $\omega_{0}$ в уравнении дисперсии (3). Эта величина определяет ширину энергетической щели в спектре колебаний деформируемого тела и отвечает почти нулевой энергии $\hbar \omega_{0}$. Это значит, что пластическая деформация всегда развивается локализовано в форме автоволновых процессов. В то же время тип реализующейся

Таблица 2. Коэффициенты в уравнении дисперсии автоволн локализованной пластичности

\begin{tabular}{|c|c|c|c|}
\hline \multirow{2}{*}{$\begin{array}{c}\text { Материал } \\
\text { (методика вариации автоволновых параметров) }\end{array}$} & $\alpha$ & $k_{0}$ & $\omega_{0}$ \\
\hline & $\mathrm{m}^{2} / \mathrm{s} \cdot 10^{2}$ & $(1 / \mathrm{m}) \cdot 10^{-3}$ & $\mathrm{~Hz} \cdot 10^{2}$ \\
\hline $\mathrm{Al}$ - поликристаллы (размер зерна) [1] & 0.79 & 1.08 & 5.5 \\
\hline Сплав на основе $\mathrm{Fe}$ - монокристаллы (ориентация) [1] & 0.54 & 1.01 & 3.6 \\
\hline Сплав на основе $\mathrm{Fe}$ - поликристаллы (температура) & 0.66 & 1.64 & 5.2 \\
\hline
\end{tabular}


автоволновой моды определяется действующим законом деформационного упрочнения.

\section{4. О температурной независимости инварианта (1)}

Обратим внимание на то обстоятельство, что величина $\chi V_{t}$, входящая в уравнение $(1)$, имеет размерность кинематической вязкости среды $\mathrm{m}^{2} / \mathrm{s}$. В этом случае нетрудно найти аналогию между инвариантом (1) и числом Рейнольдса, характеризующим режимы ламинарного и турбулентного движения жидкости [10] и записанным в виде

$$
\operatorname{Re}=\frac{l V}{v}=\frac{\rho l V}{\mu}
$$

где $l$ - характерный пространственный масштаб потока, $V$ - его скорость, $\rho$ - плотность, $v$ - кинематическая, а $\mu$ - динамическая вязкость среды. Если для случая пластического течения принять, что $l \equiv \lambda, V \equiv V_{a w}$ и $v \equiv \chi V_{t}$, то уравнение (5) совпадает с инвариантом (1).

Нужно сказать, что идея о развитии ламинарного и турбулентного режимов при пластическом течении твердых тел по аналогии с течением жидкости была высказана Коттреллом [11]. Будем считать, что в основе автоволновых механизмов пластического течения, как и в основе механизмов пластичности вообще [12], лежит движение дислокаций. Проблема их подвижности детально разработана в $[13,14]$, где показано, что скорость движения дефектов в поле напряжений контролируется вязкостью фононного газа (в металлах также и электронного [15]) и определяется соотношением

$$
V_{d i s l}=\frac{b \sigma}{B}
$$

где $B$-- константа торможения дислокаций, определяемая характеристиками фононного газа. При „деформационной“ интерпретации соотношения (5) имеет

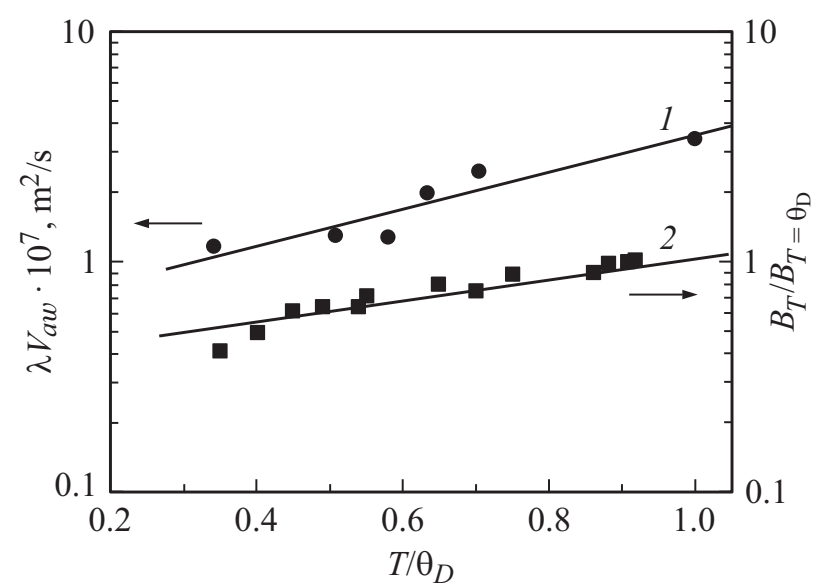

Рис. 6. Сопоставление зависимостей $\lambda V_{a w}(T)$ (1, слева) и $\frac{B}{B_{T=\theta_{D}}}(T)$ (2, справа). смысл связать вязкость $\mu$ с константой дислокационного торможения $B$ и полагать, что $\chi V_{t} \sim \mu \sim B$.

Из этого утверждения следует возможность анализа температурной зависимости критерия Рейнольдса и, соответственно, инварианта (1) на основе зависимости $B(T)$. Последняя приведена и проанализирована в работе [13]. Сопоставим показанные на рис. 6 экспериментально полученную зависимость $\lambda V_{a w}(T)$ (график 1) и зависимость $\frac{B_{T}}{B_{T=\theta_{D}}}(T)$ (график 2), построенную по данным работы [13]. В последнем соотношении $B_{T}-$ константа торможения дислокаций при температуре $T$, а $B_{T=\theta_{D}}-$ то же при $T=\theta_{D}$. Как показывает сравнение, при падении температуры испытания от $T / \theta_{D}=1$ до $T / \theta_{D}=0.3$ значения зависимостей $\lambda V_{a w}(T)$ и $\frac{B}{B_{T=\theta_{D}}}(T)$ уменьшаются почти в одинаковой степени, и выполняется отношение $\lambda V_{a w} / \chi V_{t} \approx$ const. Это можно рассматривать как указание на температурную независимость упругопластического инварианта (1).

\section{5. Дислокационное описание результатов}

При анализе связи полученных результатов с дислокационными моделями пластичности использованы данные работы [16], в которой рентгеновским методом [17] оценены плотности дислокаций $\rho$ при растяжении при температурах $143 \leq T \leq 340 \mathrm{~K}$ в сплаве, аналогичном по своему составу исследованному нами. Установлено, что для напряжения течения выполняется соотношение $\sigma=\sigma_{0}+\alpha_{0} G b \rho^{1 / 2}[12]$, где $\sigma_{0}$ и $\alpha_{0}$ - постоянные, $G-$ модуль сдвига.

На рис. 7 (прямая 1) показана зависимость напряжения течения от плотности дислокаций при растяжении, взятая из [16]. Для каждой температуры были определены значения $\rho^{1 / 2}$, соответствующие средним напряжениям течения для стадий линейного деформационного упрочнения. Поскольку стадии линейного деформационного упрочнения соответствует бегущая с постоянной скоростью $V_{a w}$ автоволна локализованного пластического течения, то можно найти корреляцию между скоростями автоволн, полученными в настоящей работе, и плотностью дислокаций, изученной в [16], в аналогичном интервале температур $143 \leq T \leq 340 \mathrm{~K}$, как показано на рис. 7 (прямая 2).

Используя дополнительно данные работы [18], согласно которым средние длины дислокационных пробегов при деформации кристаллов железа составляют $10^{-5} \leq l \leq 10^{-4} \mathrm{~m}$, а скорости движения дислокаций лежат в интервале $10^{-3} \leq V_{d i s l} \leq 10^{-2} \mathrm{~m} / \mathrm{s}$, получаем $\langle l V\rangle_{\text {disl }}=\mathrm{const} \approx 10^{-7} \mathrm{~m}^{2} / \mathrm{s}$. Постоянство этого произведения объясняется тем, что дислокации начинают двигаться, если приложенные напряжения достигают уровня внутренних напряжений, создаваемых в кристалле дислокационной сеткой $\sigma \geq \alpha_{0} G b \rho^{1 / 2}$. 


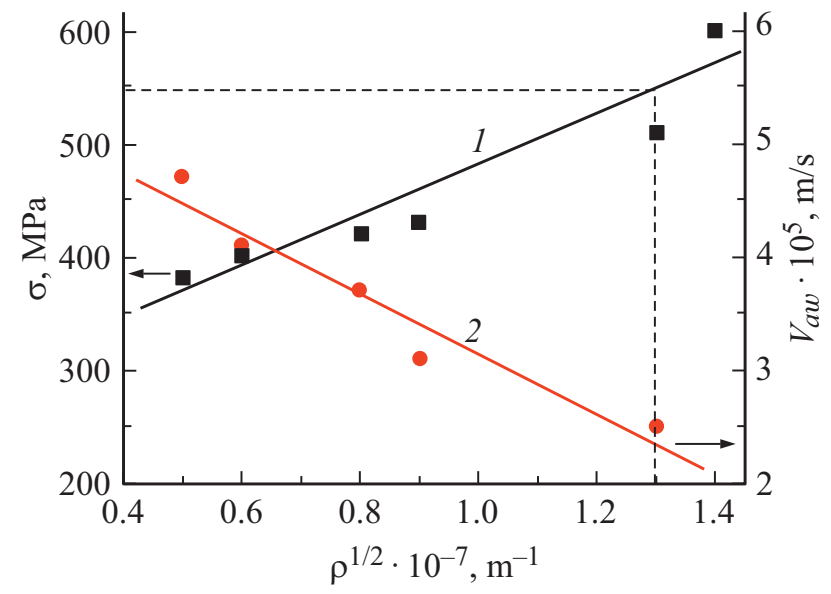

Рис. 7. Зависимость напряжения течения (1, слева) при $T=298 \mathrm{~K}[16]$ и зависимость скорости автоволн локализованной пластичности (2, справа) от плотности дислокаций по данным работы [16] в интервале температур $143 \leq T \leq 300 \mathrm{~K}$.

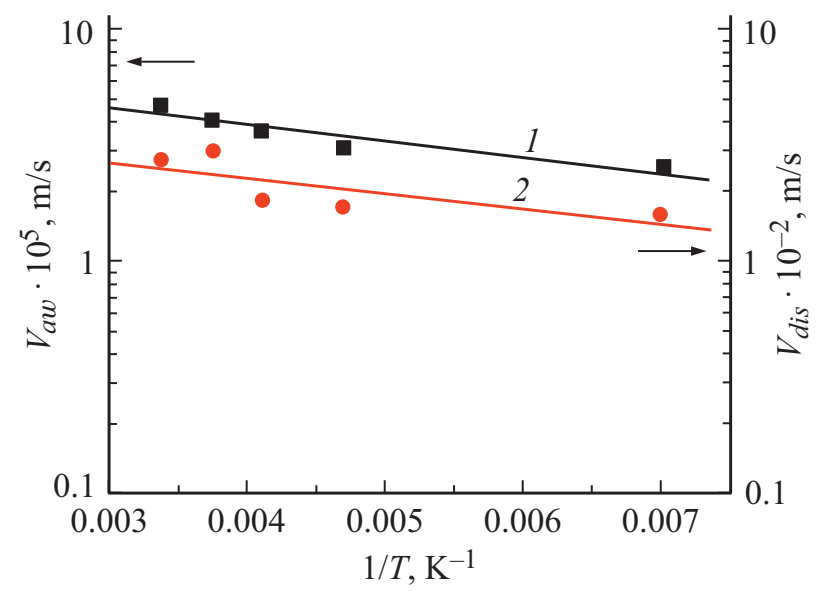

Рис. 8. Зависимость скорости автоволн локализованной от скорости движения дислокаций для стадий линейного деформационного упрочнения в интервале температур $143 \leq T \leq 300 \mathrm{~K}$.

В работах [1,2] было показано, что $\left\langle\lambda V_{a w}\right\rangle \approx\langle l V\rangle_{\text {disl }}$ $\approx 10^{-7} \mathrm{~m}^{2} / \mathrm{s}$. Тогда скорость движений дислокаций

$$
V_{\text {disl }} \approx \lambda V_{a w} l^{-1} \approx \lambda V_{a w} \rho^{1 / 2} \sim V_{a w}
$$

Очевидно, из этих данных следует корреляция скорости автоволн локализованной пластичности (макроскопическая величина) и скорости движения дислокаций (микроскопическая величина) для стадий линейного деформационного упрочнения сплава в интервале температур $143 \leq T \leq 300 \mathrm{~K}$ (рис. 8 ).

\section{4. Выводы}

1. Установлены закономерности поведения автоволновых характеристик локализованного пластического течения от температуры испытания в интервале
$143 \leq T \leq 420 \mathrm{~K}$. Показано, длина автоволны локализованной пластической деформации не зависит от температуры испытания, в то время, как скорость автоволны растет с температурой по закону Аррениуса.

2. Для области пониженных температур испытания подтверждены выполнение обратной пропорциональности скорости автоволн коэффициенту деформационного упрочнения, а также квадратичного закона дисперсии автоволн, установленных ранее для комнатной температуры.

3. Предложена интерпретация упругопластического инварианта деформации как аналога числа Рейнольдса, которая может быть перспективна и полезна для объяснения физической природы закономерностей локализованного пластического течения в материалах.

4. Предложена интерпретация температурных зависимостей параметров автоволновой пластичности на основе данных об изменениях плотности дислокаций в процессе деформирования.

\section{Благодарности}

Авторы признательны В.И. Данилову за полезные советы по методике низкотемпературных механических испытаний.

\section{Финансирование работы}

Работа выполнена в рамках государственного задания ИФПМ СО РАН, проект III.23.1.2 и частично поддержана грантом РФФИ № 20-08-00305/20.

\section{Конфликт интересов}

Авторы заявляют об отсутствии у них конфликта интересов.

\section{Список литературы}

[1] L.B. Zuev, S.A. Barannikova. Crystals 9, 458 (2019).

[2] Л.Б. Зуев. Автоволновая пластичность. Локализация и коллективные моды. Физматлит, М. (2019). 207 с.

[3] Л.Б. Зуев, С.А. Баранникова. ЖТФ 90, 773 (2020).

[4] R.E. Newnham. Properties of Materials. University Press, Oxford (2005). $378 \mathrm{p}$.

[5] R.J. Asaro, V.A. Lubarda. Mechanics of Solids and Materials. University Press, Cambridge (2006). 880 p.

[6] Р. Джоунс, К. Уайкс. Голографическая и спеклинтерферометрия. Мир, М. (1986). 327 с

[7] H.B. Huntington. The Elastic Constants of Crystals. Academic Press, N. Y. (1958). 139 p.

[8] Э. Скотт. Нелинейная наука. Рождение и развитие когерентных структур. Физматлит, М. (2007). 559 с.

[9] D. Caillard, J.L. Martin. Thermally Activated Mechanisms in Crystal Plasticity. Elsevier, Oxford (2003). 433 p.

[10] Л.Д. Ландау, Е.М. Лифшиц. Гидродинамика. Физматлит, М. (2001). 732 c. 
[11] А.Х. Коттрелл. Дислокации и пластическое течение в кристаллах. Металлургия, М. (1958). 267 с.

[12] A. Argon. Strengthening Mechanisms in Crystal Plasticity. University Press, Oxford (2008). 404 p.

[13] V.I. Al'shits, V.L. Indenbom. Dislocations in Crystals / Ed. F.R.N. Nabarro. North-Holland, Amsterdam (1986). 7, 43.

[14] D. Blaschke, E. Motolla, D.L. Preston. Phil. Mag. 100, 571 (2020).

[15] J.M. Ziman. Electrons and Phonons. University Press, Oxford (2001). 576 p.

[16] J. Talonen, P. Nenonen, G. Pape, H. Hänninen. Met. Mater. Trans. A 36, 421 (2005).

[17] M. Wilkens. Phys. Status Solidi A 2, 359 (1970).

[18] D. Caillard. Acta Mater. 58, 3493 (2010).

Редактор К.В. Емцев 